Cerebrovasc Dis 2010;29:408-409

DOI: $10.1159 / 000288055$

\section{Positional Brain Ischemia with MCA Occlusion Successfully Treated with Extra-Intracranial Bypass}

\author{
A. Albanese a , G. Esposito a , A. Puca a , A. Tuttolomondo d, \\ B. Tirpakova ${ }^{\text {a }}$ D. Di Giuda ', G. Maira ${ }^{\text {a }}$, V. Di Lazzaro ${ }^{\text {b }}$ \\ Institutes of a Neurosurgery, ${ }^{\mathrm{b}}$ Neurology and ${ }^{\mathrm{c}}$ Nuclear Medicine, \\ Catholic University, Rome, and ${ }^{\mathrm{d}}$ Biomedical Department \\ of Internal and Specialist Medicine, University of Palermo, \\ Palermo, Italy
}

Positional cerebral ischemia (PCI) is an extremely rare condition in which dramatic central nervous system deficits are triggered by standing in patients with severe stenosis or occlusion of the major brain-supplying blood vessels [1]. Because PCI is generally associated with orthostatic hypotension $[2,3]$, a hemodynamic mechanism has been proposed [4]. However, PCI has been reported even in patients without orthostatic hypotension [1]. The treatment is medical, but surgery can be considered in refractory cases (endarterectomy for carotid artery stenosis or either high- or low-flow extra-intracranial bypass - for carotid occlusion) [3]. We report a case of PCI associated with middle cerebral artery (MCA) occlusion and without orthostatic hypotension treated by lowflow extra-intracranial bypass.

The patient, a 58 -year-old male with a history of hypertension, suffered an ischemic stroke in the left hemisphere with sudden onset of speech disturbance and right hemiparesis in $1992 . \mathrm{He}$ experienced complete neurological recovery in approximately 1 month. Aspirin was the medical treatment. In December 2004, while the patient was still on aspirin, he presented with several stereotyped TIAs with acute onset of right-sided hemiparesis and paresthesias, and mild motor aphasia. Symptoms that were always triggered by standing presented nearly daily and rapidly improved (within few minutes) on returning to the supine position. Brain MRI showed multiple chronic ischemic lesions in the territory of the left MCA. Dual antiplatelet therapy with clopidogrel plus aspirin was started, followed by reduction in the frequency of the TIAs. In May 2005, recurrent episodes of right hemiparesis were associated with incomplete recovery when lying down, and he was hospitalized. General examination was normal and there were no hematological abnormalities. The supine blood pressure ranged between 130/80 and 150/90 mm Hg. There was no significant orthostatic hypotension and only occasionally was a slight reduction $(<10 \mathrm{~mm} \mathrm{Hg})$ in systolic blood pressure observed on standing. However, just a few blood pressure evaluations were performed on standing because of the worsening of neurological symptoms in this condition. MRI showed multiple ischemic areas in the territory of the left MCA (fig. 1A), whereas MR angiography demonstrated the occlusion of the left MCA (fig. 1B). A cerebral angiogram confirmed the occlusion of the left MCA at the M1 segment and a hypovascularized area in the left parietal region (fig. 1C). SPECT scans revealed a severe impairment of regional cerebral perfusion throughout the left hemisphere involving both deep and superficial territories; a marked reduction in ${ }^{99 \mathrm{~m}} \mathrm{Tc}$ ECD uptake was detected in the frontal and temporal cortices, while absence of radiotracer uptake was found in the parietal and occipital cortices (fig. 1E, F). Screening for vasculitis, thrombophilic pathologies and cardioembolic conditions were negative. There was a nonsignificant stenosis (40\%) of the left internal carotid. Despite dual antiplatelet therapy the patient progressively worsened with symptoms manifesting themselves also while he was lying supine. Because of the progression of neurological symptoms and the evidence of left MCA occlusion on angiography, it was decided to perform an extra-intracranial bypass. This was performed by anastomoses between 2 distal branches of the left superficial temporal artery (STA) and 2 cortical posterior frontal and parietal branches of the left MCA. The postoperative course was characterized by a progressive neurological improvement. The patient tolerated the sitting position on the second postoperative day and was able to stand up after a week.

The bypass was patent on cerebral angiogram 2 weeks after surgery. A SPECT evaluation carried out 5 months after surgery showed a marked improvement in regional cerebral perfusion in the left cortical regions (fig. 1G, H). An angiogram (after 8 months) demonstrated the patency of the bypass and the presence of a reperfusion area in the left posterior parietal region (fig. 1D). At the last clinical control (after 3 years), the patient did not report any further cerebrovascular symptoms, and his daily living activities were normal (with no concern for standing and walking).

The patient we describe appears to be the first published example of PCI associated with MCA occlusion and without significant orthostatic hypotension, successfully treated by STAMCA bypass.

\section{References}

1 Caplan LR, Sergay S: Positional cerebral ischaemia. J Neurology Neurosurg Psychiatry 1976;39:385-391.

2 Marti-Fabregas J, Cocho D, Lleo A, Marti-Vilalta JL: Transcranial Doppler recording in a patient with transient positional cerebral ischemia. Neurology 2000;55:731-732.

-3 Melgar MA, Sahni D, Weinand M: Thyrocervical trunk-external carotid artery bypass for positional cerebral ischemia due to common carotid artery occlusion. Technical note. J Neurosurg 2005; 103:170175.

4 Dobkin BH: Orthostatic hypotension as a risk factor for symptomatic occlusive cerebrovascular disease. Neurology 1989;39:30-34.

Vincenzo Di Lazzaro

Istituto di Neurologia, Università Cattolica

Largo A. Gemelli 8

IT-00168 Rome (Italy)

Tel. +39063015 4435, Fax +39063550 1909

E-Mail vdilazzaro@rm.unicatt.it 

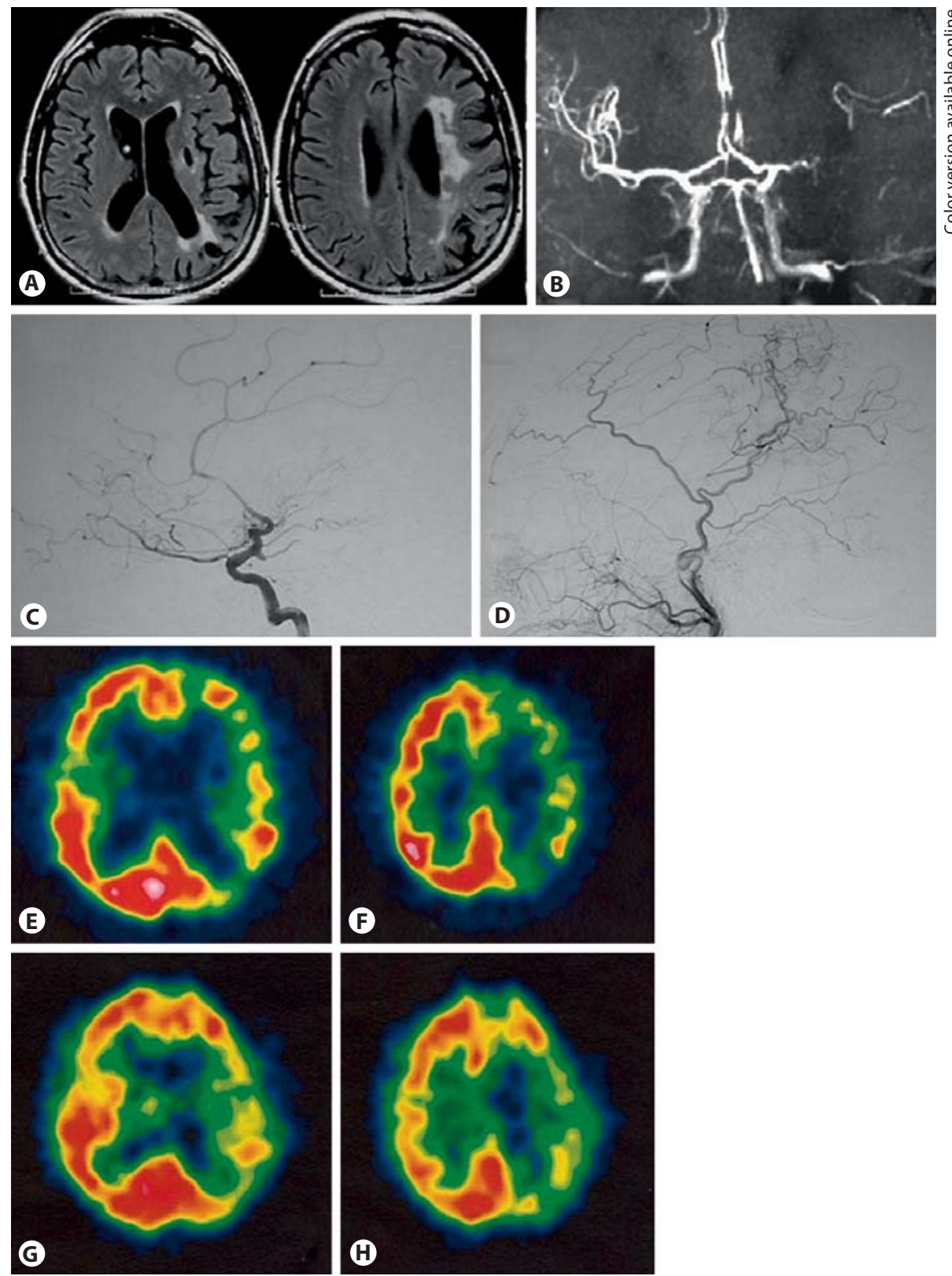

Fig. 1. A Axial FLAIR images showing an ovally shaped malacic lesion and diffuse left periventricular ischemic areas. B MR angiography of obstruction of the left MCA. C Presurgical cerebral angiogram showing occlusion of the left MCA and parietal hypovascularized area. D Postoperative external carotid artery angiogram illustrating bypass patency and revascularized parietal areas. E, F Presurgical SPECT images showed absent ${ }^{99 \mathrm{~m} T c \text { ECD }}$

uptake (cold area) in the left parietal and occipital cortices. Decreased radiotracer uptake (hypoperfusion) was seen in the left frontal and temporal regions. G, $\mathbf{H}$ In the SPECT study carried out 5 months after surgery, a marked increase in radiotracer uptake in the parietal and occipital cortices as well as the frontal and temporal regions was found. 